\title{
SCALABLE LOCALIZED MODEL ORDER REDUCTION APPLIED TO COMPOSITE AERO-STRUCTURES
}

\author{
J. Bénézech ${ }^{1 *}$, L. Seelinger ${ }^{2}$, T.J. Dodwell ${ }^{3,4}$, P. Bastian ${ }^{2}$, R. Scheichl ${ }^{2}$ and R. Butler $^{1}$ \\ ${ }^{1}$ Material \& Structure Research Centre, University of Bath, Bath, UK; ${ }^{2}$ Interdisciplinary Center for \\ Scientific Computing and Institute for Applied Mathematics, Heidelberg University, Heidelberg, \\ Germany; ${ }^{3}$ Institute of Data Science and AI, University of Exeter, Exeter, UK; ${ }^{4}$ The Alan Turing \\ Institute, The British Library, London, UK. \\ *jb3285@bath.ac.uk
}

The CerTest project aims at developing a new design/certification process, adapted to composites for aerospace application. The quantification of uncertainties, arising from material variability and experimental measurements for example, forms a critical challenge. When applied to large scale composite parts, the primary challenge is the cost of the associated numerical simulation required to evaluate the material response for a (typically large) set of parameters. To this end, a GMsFEM [1] type method has been chosen to efficiently simulate large parts (up to a billion dofs) without scale separation, illustrated on Figure 1. Variants of GMsFEM differ in their choice of local basis: a suitable choice for structural mechanics is the one derived from the Generalized Eigenvalue problem for Overlapping subdomains (GenEO) [2]. To be integrated in a stochastic framework the computational cost is divided into two phases: offline and online. In the offline phase, the GenEO coarse space is generated using a parallel setting for a given set of parameters (i.e. a pristine part). During this phase, information is stored; a database is hence initiated. The online phase is dedicated to assess the effect of one or multiple changes in the parameters (such as a defect). This offers huge computational savings for large components, since the majority of basis functions are simply loaded from the database. Thus, online phases can be carried out on single processors, freeing parallel compute resources to sample the parameters space.

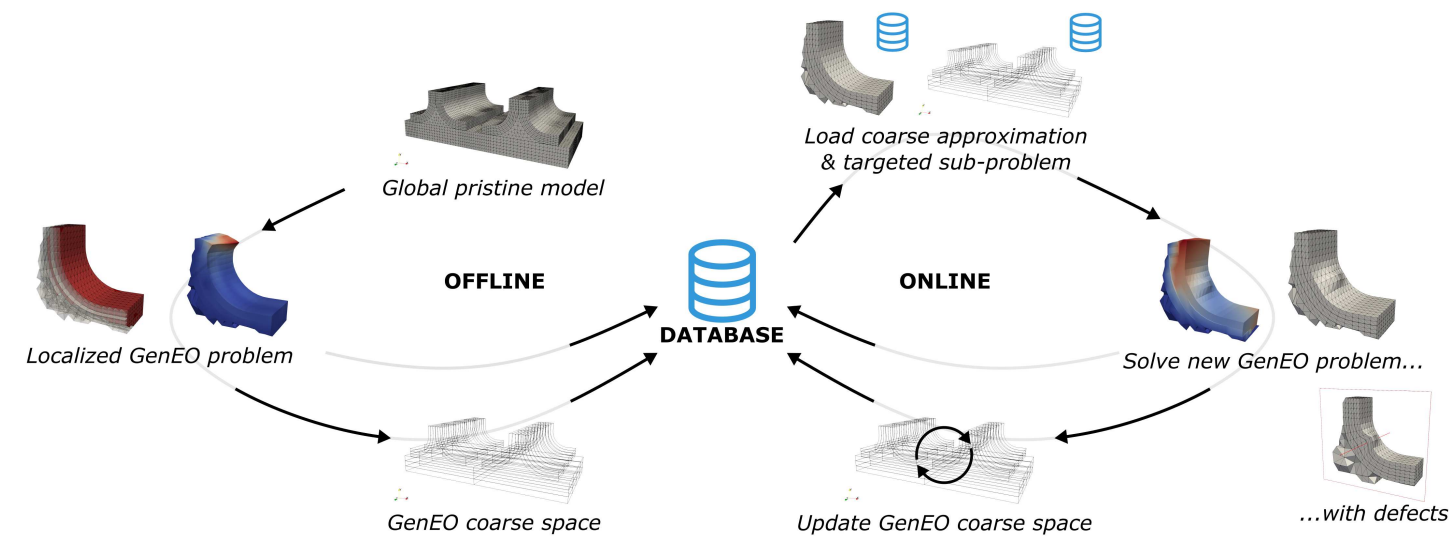

Figure 1. Schematic of the Offline/Online approach.

[1] Y. Efendiev, J. Galvis and T.Y. Hou (2013) Generalized multiscale finite element methods (GMsFEM). Journal of Computational Physics, 251, 116-135.

[2] A. Reinarz, T.J. Dodwell, T. Fletcher, L. Seelinger, R. Butler and R. Scheichl (2018) Dune-composites-A new framework for high-performance finite element modelling of laminates. Composite Structures, 184, 269-278. 